\title{
Local readout enhancement for detuned signal-recycling interferometers
}

\author{
Henning Rehbein, ${ }^{1}$ Helge Müller-Ebhardt, ${ }^{1}$ Kentaro Somiya, ${ }^{2}$ Chao Li ${ }^{3}$ Roman Schnabel, ${ }^{1}$ \\ Karsten Danzmann, ${ }^{1}$ and Yanbei Chen ${ }^{2}$ \\ ${ }^{1}$ Max-Planck-Institut für Gravitationsphysik (Albert-Einstein-Institut), Institut für Gravitationsphysik, Leibniz Universität Hannover, \\ Callinstr. 38, 30167 Hannover, Germany \\ ${ }^{2}$ Max-Planck-Institut für Gravitationsphysik (Albert-Einstein-Institut), Am Mühlenberg 1, 14476 Potsdam, Germany \\ ${ }^{3}$ California Institute of Technology, M/C 130-33, Pasadena, California 91125, USA
}

(Received 21 May 2007; published 21 September 2007)

\begin{abstract}
High power detuned signal-recycling interferometers currently planned for second-generation interferometric gravitational-wave detectors (for example Advanced LIGO) are characterized by two resonances in the detection band, an optical resonance and an optomechanical resonance which is upshifted from the suspension pendulum frequency due to the so-called optical-spring effect. The detector's sensitivity is enhanced around these two resonances. However, at frequencies below the optomechanical resonance frequency, the sensitivity of such interferometers is significantly lower than non-optical-spring configurations with comparable circulating power; such a drawback can also compromise high-frequency sensitivity, when an optimization is performed on the overall sensitivity of the interferometer to a class of sources. In this paper, we clarify the reason for such a low sensitivity, and propose a way to fix this problem. Motivated by the optical-bar scheme of Braginsky, Gorodetsky, and Khalili, we propose to add a local readout scheme which measures the motion of the arm-cavity front mirror, which at low frequencies moves together with the arm-cavity end mirror, under the influence of gravitational waves. This scheme improves the low-frequency quantum-noise-limited sensitivity of optical-spring interferometers significantly and can be considered as an incorporation of the optical-bar scheme into currently planned secondgeneration interferometers. On the other hand it can be regarded as an extension of the optical-bar scheme. Taking compact binary inspiral signals as an example, we illustrate how this scheme can be used to improve the sensitivity of the planned Advanced LIGO interferometer, in various scenarios, using a realistic classical-noise budget. We also discuss how this scheme can be implemented in Advanced LIGO with relative ease.
\end{abstract}

DOI: 10.1103/PhysRevD.76.062002

PACS numbers: 04.80.Nn, 03.65.Ta, 42.50.Dv, 42.50.Lc

\section{INTRODUCTION}

First-generation laser interferometric gravitational-wave (GW) detectors (LIGO [1], VIRGO [2], GEO [3], and TAMA [4]) are reaching design sensitivities. These interferometers are usually Michelson interferometers with Fabry-Perot cavities in the arms, with power-recycling (PR) at the laser input port (with the exception of GEO, which uses dual-recycling [5]), and operating close to the dark-port condition.

In order to have a flexible sensitivity to specific astrophysical sources, and for other technical reasons such as lowering power at the beam splitter (BS), secondgeneration interferometers, such as Advanced LIGO [6], plan to use the so-called signal-recycling (SR) configuration, in which an additional mirror is placed at the dark port of a Fabry-Perot Michelson interferometer, modifying the optical resonant structure of the interferometer. The adjustment of the location and reflectivity of the signal-recycling mirror varies the optical resonance frequency and bandwidth, respectively. Near the optical resonance, sensitivity to GWs is improved. When the signal-recycling cavity, the cavity formed by the input test-mass mirrors and the signal-recycling mirror is neither resonant nor antiresonant with respect to the carrier frequency, the optical configuration is called detuned signal-recycling. In these detuned configurations, the optical resonance of the interferometer is away from the carrier frequency, creating a peak sensitivity to GWs away from DC.

As demonstrated theoretically by Buonanno and Chen [7-9] and experimentally by Somiya et al. [10] and Miyakawa et al. [11], detuned signal-recycling also makes the power inside the interferometer depend on the motion of the mirrors, creating an optical spring, and can shift the eigenfrequency of the test masses from the pendulum frequency $(\sim 1 \mathrm{~Hz})$ up to the detection band. The optical spring helps to improve the interferometer's response to GWs around the optomechanical resonant frequency, even allowing the interferometer to surpass the free-mass standard quantum limit (SQL). However, the quantum-noiselimited sensitivity of optical-spring interferometers at frequencies below the optomechanical resonant frequency is dramatically lower than the one of non-optical-spring interferometers. Such a limitation in sensitivity is caused by the optical spring, which rigidly connects the front and the end mirror of the arm cavities at frequencies below the optomechanical resonance. The general principle underlying this effect has already been explained in the works of Braginsky, Gorodetsky, and Khalili, namely, in their proposal of the optical-bar detection scheme [12]. In order to understand this more conveniently, we need to use the local inertial frame of the BS, in which the effect of GWs can be 
described completely as a tidal force field, which induces forces only on the end test-mass mirrors (ETMs), but not on the input test-mass mirrors (ITMs). We make the approximation that the ITMs and the BS are colocated. In this frame, the propagation of the light is unaffected by GWs. Remember that the optical spring connects the ITM and the ETM. At frequencies substantially below the optomechanical resonance, the optical spring behaves like a rigid optical bar, connecting the ITM and the ETM of each arm rigidly. It is then easy to understand that the carrier light, which senses the change in arm-cavity length, or the difference in ITM and ETM motion, cannot be used to measure GWs efficiently at these frequencies. On the other hand, since the ITM and the ETM are rigidly connected, they both move, in the local inertial frame of the BS, by $1 / 2$ the amount the ETM would have moved if there were no optical spring present (assuming ITM and ETM to have equal masses). To illustrate this situation, assume that a low-frequency GW with amplitude $h$ is incident from right above our detector (with arm length $L$ ), then in the local inertial frame of the BS, the motion of the ETM of a nonoptical-spring interferometer would be $L h$, the motion of ITM and ETM of an optical-spring interferometer below resonance will both be $\sim L h / 2$. For this reason, if one also measures the local motion of the ITM using an additional local readout scheme, one can recover low-frequency sensitivity dramatically. Note that as viewed by the local meter, the ITM has an effective mass that is equal to the total mass of the ITM and the ETM. If one applies a local readout scheme to the ETM, the same sensitivity recovery is possible, since the ETM also moves with respect to a free colocated mirror by $-L h / 2$. Braginsky, Gorodetsky, and Khalili proposed an optical-bar detection scheme, in which only the local motion of the ITM is measured [12]. In this sense, what we are proposing can be considered as directly incorporating the optical-bar scheme into currently planned second-generation interferometers.

Local readout schemes have also been proposed for interferometers without optical spring, with a different motivation. In those interferometers, the motion of mirrors with respect to their local inertial frames are caused by radiation-pressure noise (if we only consider signal and quantum noise sources); results of local readout schemes can thus be used to cancel radiation-pressure noise and improve low-frequency sensitivity [13,14]. Furthermore, such schemes are able to cancel parts of the classical noise. Our treatment here can also be viewed as a generalization of these schemes because by setting detuning in our treatment to zero, we will recover their results.

From an astrophysical point of view, the addition of the local readout scheme, which broadens the detection band, will allow the interferometer to search for multiple sources simultaneously, as well as to examine a wider frequency range of the same source. As an example, we will explore how the increase in detection bandwidth can allow us to

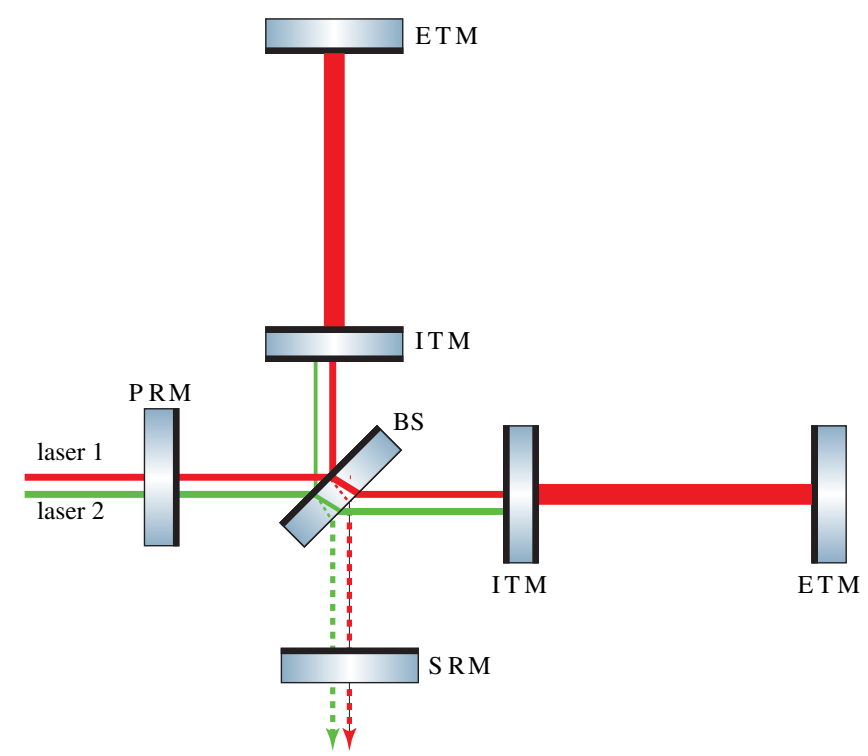

FIG. 1 (color online). Schematic plot of a power- and signalrecycled Michelson interferometer with arm cavities and doublereadout. The added local readout sensing the ITM is realized by a secondary laser which does not resonate in the arm cavities.

detect more efficiently the population of compact binary objects with a broad range of masses (and hence signal frequency band).

In order to construct the local meter, we consider a scheme where a second carrier is injected into the bright port, which does not enter the arm cavities, but instead senses the location of the ITMs, as shown in Fig. 1. An alternative strategy would be attaching auxiliary interferometers at the ETMs. These two strategies are quite equivalent in the ideal situation, but differ from each other in terms of difficulty in implementation, in terms of quantum noise, and in terms of technical noise sources such as laser noise as we will discuss in some more detail.

This paper is organized as follows. In Sec. II, we study the dynamics, sensing, and control of our double-readout interferometer. In Sec. II A, we write down and solve the joint Heisenberg equations of motion of test masses, beam splitter, and optical fields; in Sec. II B, we evaluate the optimal combined GW sensitivity of the two readout channels; in Sec. II C, we prove that the use of control schemes does not affect this sensitivity. In Sec. IV we show the benefit which the local readout scheme will provide for the detection of intermediate-mass black holes, using a realistic Advanced LIGO noise budget. In Sec. IV we consider practical issues for a possible implementation in Advanced LIGO. In Sec. V we summarize our main conclusions.

\section{DYNAMICS, SENSING AND CONTROL}

\section{A. Equations of motion}

Let us consider a configuration where the ITM motion of a signal- and power-recycled Michelson interferometer 
with arm cavities is locally sensed by a small interferometer which has the ITMs as its end mirrors (cf. Fig. 1). This is realized by injecting a second carrier into the bright port, which does not resonate in the arms (preferably antiresonant). Because the frequency (and the polarization) of the second carrier is (are) different from that of the first, we effectively obtain a two interferometers in one scheme where parameters such as detuning and mirror reflectivities for each interferometer can be chosen independently; input vacuum fluctuations associated with the two lasers are also independent.

Throughout this paper, we will assume the GW with amplitude $h$ as incident from right above the interferometer, with a polarization that maximizes the response of our $L$-shaped Michelson interferometers. In the following we will list the Heisenberg equations of motion in the frequency domain $[7-9,15,16]$ for the differential mode of motion (i.e., opposite in the two arms) of the input mirrors $\hat{x}_{\text {ITM }}$ and the end mirrors $\hat{x}_{\mathrm{ETM}}$, respectively, as well as for the BS motion normal to its reflective surface $\hat{x}_{\mathrm{BS}}$ and for the two measurement outputs $\hat{y}^{(i)}$ :

$$
\begin{aligned}
\hat{x}_{\mathrm{ITM}}= & -R_{x x}(\Omega)\left[\hat{F}^{(1)}(\Omega)+R_{F F}^{(1)}(\Omega)\left(\hat{x}_{\mathrm{ETM}}-\hat{x}_{\mathrm{ITM}}\right)\right. \\
& \left.-\hat{F}^{(2)}(\Omega)-R_{F F}^{(2)}(\Omega)\left(\hat{x}_{\mathrm{ITM}}+\sqrt{2} \hat{x}_{\mathrm{BS}}\right)\right]+\hat{\xi}_{\mathrm{ITM}},
\end{aligned}
$$

$$
\begin{aligned}
& \hat{x}_{\mathrm{ETM}}=R_{x x}(\Omega)\left[\hat{F}^{(1)}(\Omega)+R_{F F}^{(1)}(\Omega)\left(\hat{x}_{\mathrm{ETM}}-\hat{x}_{\mathrm{ITM}}\right)\right] \\
&+ L h+\hat{\xi}_{\mathrm{ETM}}, \\
& \hat{x}_{\mathrm{BS}}= R_{x x}^{\mathrm{BS}}(\Omega)\left[\hat{F}^{(2)}(\Omega)+R_{F F}^{(2)}(\Omega)\left(\hat{x}_{\mathrm{ITM}}+\sqrt{2} \hat{x}_{\mathrm{BS}}\right)\right. \\
&+\left.\hat{F}_{\mathrm{BP}}^{(1)}(\Omega)+\hat{F}_{\mathrm{BP}}^{(2)}(\Omega)\right]+\hat{\xi}_{\mathrm{BS}}, \\
& \hat{y}^{(1)}= \hat{Y}_{1}^{(1)}(\Omega) \sin \zeta^{(1)}+\hat{Y}_{2}^{(1)}(\Omega) \cos \zeta^{(1)} \\
&+\left[R_{Y_{1} F}^{(1)}(\Omega) \sin \zeta^{(1)}+R_{Y_{2} F}^{(1)}(\Omega) \cos \zeta^{(1)}\right] \\
& \times\left(\hat{x}_{\mathrm{ETM}}-\hat{x}_{\mathrm{ITM}}\right), \\
& \hat{y}^{(2)}= \hat{Y}_{1}^{(2)}(\Omega) \sin \zeta^{(2)}+\hat{Y}_{2}^{(2)}(\Omega) \cos \zeta^{(2)} \\
&+\left[R_{Y_{1} F}^{(2)}(\Omega) \sin \zeta^{(2)}+R_{Y_{2} F}^{(2)}(\Omega) \cos \zeta^{(2)}\right] \\
& \times\left(\hat{x}_{\mathrm{ITM}}+\sqrt{2} \hat{x}_{\mathrm{BS}}\right) .
\end{aligned}
$$

Note that $\hat{x}_{\text {ITM }}$ and $\hat{x}_{\text {ETM }}$ account for the differential motion between two mirrors while $\hat{x}_{\mathrm{BS}}$ describes the motion of a single mirror with an angle of $45 \mathrm{deg}$. This explains the factor of $\sqrt{2}$ in front of the BS motion. The out-going fields at the dark port belonging to the two different carriers are each sensed by homodyne detection such that the measurement outputs are a certain combination of amplitude and phase quadratures (described by the phases $\zeta^{(1),(2)}$ ). Note that we have labeled all quantities with superscripts (1) and (2) for the large-scale interferometer and the local meter (the small interferometer, formed by the BS and the ITMs), respectively. The operators $\hat{F}^{(i)}$ and $\hat{F}_{\mathrm{BP}}^{(i)}$ describe the radiation-pressure forces which would act on fixed mirrors caused by the incoming vacuum fields at the dark port and the laser light fluctuations from the bright port, respectively. The operators $\hat{Y}_{j}^{(i)}$ account for the shot noise in the case of fixed mirrors. Each optical component is subject to classical noise generated by the corresponding operator $\hat{\xi}$ and has its own mechanical susceptibility $R_{x x}$. The susceptibilities $R_{F F}^{(i)}$ describe the optical springs [7] and $R_{Y_{i} F}^{(i)}$ the transformation of the mirror motion into the two outputs. In the following we will present all these quantities in more detail while all appearing parameters are summarized in Table I.

The free radiation-pressure force and the free shot noise in each of the two interferometers are given by [9]

$$
\begin{aligned}
& \hat{F}^{(i)}=\sqrt{\frac{\epsilon^{(i)} \theta^{(i)} m \hbar}{2}} \frac{\left(\mathrm{i} \Omega-\epsilon^{(i)}\right) \hat{a}_{1}^{(i)}+\lambda^{(i)} \hat{a}_{2}^{(i)}}{\left(\Omega-\lambda^{(i)}+\mathrm{i} \epsilon^{(i)}\right)\left(\Omega+\lambda^{(i)}+\mathrm{i} \epsilon^{(i)}\right)}, \\
& \hat{Y}_{1}^{(i)}=\frac{\left(\left(\lambda^{(i)}\right)^{2}-\left(\epsilon^{(i)}\right)^{2}-\Omega^{2}\right) \hat{a}_{1}^{(i)}+2 \lambda^{(i)} \epsilon^{(i)} \hat{a}_{2}^{(i)}}{\left(\Omega-\lambda^{(i)}+\mathrm{i} \epsilon^{(i)}\right)\left(\Omega+\lambda^{(i)}+\mathrm{i} \epsilon^{(i)}\right)}, \\
& \hat{Y}_{2}^{(i)}=\frac{-2 \lambda^{(i)} \epsilon^{(i)} \hat{a}_{1}^{(i)}+\left(\left(\lambda^{(i)}\right)^{2}-\left(\epsilon^{(i)}\right)^{2}-\Omega^{2}\right) \hat{a}_{2}^{(i)}}{\left(\Omega-\lambda^{(i)}+\mathrm{i} \epsilon^{(i)}\right)\left(\Omega+\lambda^{(i)}+\mathrm{i} \epsilon^{(i)}\right)},
\end{aligned}
$$

where $\theta^{(i)}=\frac{8 P^{(i)} \omega_{0}^{(i)}}{m L^{(i)} c}$ has units of frequency cubed. Note that $P^{(i)}$ refers to the circulating power in each arm, respectively. Here $\hat{a}_{1}^{(i)}$ and $\hat{a}_{2}^{(i)}$ are the amplitude and phase quadrature operators of the incoming vacuum fields at the dark port [15], associated with the first and second

TABLE I. Technical data and parameter values for large-scale interferometer and local meter used throughout the calculations. Note that we have defined $\rho_{\mathrm{SR}}$ with respect to the first carrier while the local meter requires a different reflectivity in order to achieve the bandwidth $\epsilon^{(2)}$.

\begin{tabular}{lcc}
\hline \hline Symbol & physical meaning & value \\
\hline$m$ & single mirror mass & $40 \mathrm{~kg}$ \\
$m_{\mathrm{BS}}$ & beam splitter mass & $40 \mathrm{~kg}$ \\
$c / \omega_{0}^{(1)}$ & laser wavelength of 1st carrier & $1064 \mathrm{~nm}$ \\
$P^{(1)}$ & circulating power of 1st carrier & $0.1 \ldots 0.8 \mathrm{MW}$ \\
$L^{(1)}$ & large-scale interferometer arm length & $4 \mathrm{~km}$ \\
$\rho_{\mathrm{PR}}$ & power-recycling mirror reflectivity & $\sqrt{0.94}$ \\
$\phi$ & detuning phase for 1st carrier & $0 \ldots \pi$ \\
$\rho_{\mathrm{SR}}$ & signal-recycling mirror reflectivity & $\sqrt{0.93}$ \\
$\gamma_{o}$ & cavity half bandwidth for 1st carrier & $2 \pi 15 \mathrm{~Hz}$ \\
$\zeta^{(1)}$ & detection angle for 1st carrier & $0 \ldots \pi$ \\
$c / \omega_{0}^{(2)}$ & laser wavelength of 2nd carrier & $1064 \mathrm{~nm}$ \\
$P^{(2)}$ & circulating power of 2nd carrier & $0 \ldots 16 \mathrm{~kW}$ \\
$L^{(2)}$ & local meter arm length & $15 \mathrm{~m}$ \\
$\lambda^{(2)}$ & detuning for 2nd carrier & $0 \mathrm{~Hz}$ \\
$\epsilon^{(2)}$ & cavity half bandwidth for 2nd carrier & $2 \pi 4 \mathrm{kHz}$ \\
$\zeta^{(2)}$ & detection angle for 2nd carrier & 0 \\
\hline \hline
\end{tabular}


carrier field, respectively. The susceptibilities are given by [9]

$$
\begin{aligned}
R_{x x}^{\mathrm{BS}} & =-\frac{\sqrt{2}}{m_{\mathrm{BS}} \Omega^{2}}, \\
R_{x x} & =-\frac{2}{m \Omega^{2}}, \\
R_{F F}^{(i)} & =\frac{\theta^{(i)} m}{4} \frac{\lambda^{(i)}}{\left(\Omega-\lambda^{(i)}+\mathrm{i} \epsilon^{(i)}\right)\left(\Omega+\lambda^{(i)}+\mathrm{i} \epsilon^{(i)}\right)}, \\
R_{Y_{1} F}^{(i)} & =\sqrt{\frac{\epsilon^{(i)} \theta^{(i)} m}{2 \hbar} \frac{\lambda^{(i)}}{\left(\Omega-\lambda^{(i)}+\mathrm{i} \epsilon^{(i)}\right)\left(\Omega+\lambda^{(i)}+\mathrm{i} \epsilon^{(i)}\right)},} \\
R_{Y_{2} F}^{(i)} & =-\sqrt{\frac{\epsilon^{(i)} \theta^{(i)} m}{2 \hbar}} \frac{\epsilon^{(i)}-\mathrm{i} \Omega}{\left(\Omega-\lambda^{(i)}+\mathrm{i} \epsilon^{(i)}\right)\left(\Omega+\lambda^{(i)}+\mathrm{i} \epsilon^{(i)}\right)},
\end{aligned}
$$

where the (free) optical resonant frequency of the largescale interferometer at $\Omega=-\lambda^{(1)}-\mathrm{i} \epsilon^{(1)}$ is determined by

$$
\begin{aligned}
& \lambda^{(1)}=\gamma_{o} \frac{2 \rho_{\mathrm{SR}} \sin (2 \phi)}{1+\rho_{\mathrm{SR}}^{2}+2 \rho_{\mathrm{SR}} \cos (2 \phi)}, \\
& \epsilon^{(1)}=\gamma_{o} \frac{1-\rho_{\mathrm{SR}}^{2}}{1+\rho_{\mathrm{SR}}^{2}+2 \rho_{\mathrm{SR}} \cos (2 \phi)} .
\end{aligned}
$$

As already mentioned, the second carrier does not resonate in the arm cavities and therefore the local meter is just equivalent to an interferometer configuration without cavities in the arms. Thus, in Eq. (3) we only take into account the forces on the BS due to field fluctuations around the second carrier, in the same way as in Ref. [17]: the first two terms in the bracket on the right-hand side of Eq. (3) are due to dark-port fluctuations around the second carrier, while the third and fourth term, given by

$$
\begin{aligned}
& \hat{F}_{\mathrm{BP}}^{(1)}=\gamma_{0} \frac{L^{(1)} \sqrt{\theta^{(1)} m \hbar\left(1-\rho_{\mathrm{PR}}^{2}\right) \gamma_{0}}}{\sqrt{2} c\left(-\gamma_{0}\left(1-\rho_{\mathrm{PR}}\right)+\mathrm{i}\left(1+\rho_{\mathrm{PR}}\right) \Omega\right)} b_{1}^{(1)}, \\
& \hat{F}_{\mathrm{BP}}^{(2)}=\sqrt{\frac{\theta^{(2)} m L^{(2)} \hbar\left(1+\rho_{\mathrm{PR}}\right)}{2 c\left(1-\rho_{\mathrm{PR}}\right)} b_{1}^{(2)},}
\end{aligned}
$$

are forces due to bright-port fluctuations, where $b_{1}^{(i)}$ are the amplitude quadrature of fluctuations around the first and second carrier, at the input port. Forces due to fluctuations around the first carrier are usually negligible, because the intensity of the first carrier at the beam splitter is lower than that of the second carrier; in addition, fluctuations associated with the first carrier also do not build up as much as those associated with the second carrier, both in common and in differential mode.

In Eq. (4), we make the approximation that the first carrier only senses the cavity length, $\hat{x}_{\mathrm{ETM}}-\hat{x}_{\mathrm{ITM}}$, ignoring the slight difference between its sensitivities to ITM and ETM, as well as motion of the BS. In Eq. (5), the second carrier only senses the ITM and BS motions, since it does not enter the arm cavities.
The operators $\hat{\xi}_{\text {ITM }}, \hat{\xi}_{\mathrm{ETM}}$, and $\hat{\xi}_{\mathrm{BS}}$ model the classical noise at ITM, ETM, and BS, respectively. We assume that they are uncorrelated but all have the same spectrum, namely, one fourth of the classical-noise spectrum generally expected for the differential mode of motion. By using the following only nonvanishing correlation functions,

$$
\begin{aligned}
&\left\langle\hat{a}_{k}^{(i)}(\Omega)\left(\hat{a}_{l}^{(j)}\right)^{\dagger}\left(\Omega^{\prime}\right)\right\rangle_{\mathrm{sym}}=\pi \delta\left(\Omega-\Omega^{\prime}\right) \delta_{i j} \delta_{k l}, \\
&\left\langle\hat{b}_{k}^{(i)}(\Omega)\left(\hat{b}_{l}^{(i)}\right)^{\dagger}\left(\Omega^{\prime}\right)\right\rangle_{\mathrm{sym}}=\pi \delta\left(\Omega-\Omega^{\prime}\right) \delta_{k l} S_{l}^{(i)}(\Omega), \\
&\left\langle\hat{\xi}_{\mathrm{ITM}}(\Omega)\left(\hat{\xi}_{\mathrm{ITM}}\right)^{\dagger}\left(\Omega^{\prime}\right)\right\rangle_{\mathrm{sym}}=2 \pi \delta\left(\Omega-\Omega^{\prime}\right) S_{\mathrm{cl}}(\Omega), \\
&\left\langle\hat{\xi}_{\mathrm{ETM}}(\Omega)\left(\hat{\xi}_{\mathrm{ETM}}\right)^{\dagger}\left(\Omega^{\prime}\right)\right\rangle_{\mathrm{sym}}=2 \pi \delta\left(\Omega-\Omega^{\prime}\right) S_{\mathrm{cl}}(\Omega), \\
&\left\langle\hat{\xi}_{\mathrm{BS}}(\Omega)\left(\hat{\xi}_{\mathrm{BS}}\right)^{\dagger}\left(\Omega^{\prime}\right)\right\rangle_{\mathrm{sym}}=\pi \delta\left(\Omega-\Omega^{\prime}\right) S_{\mathrm{cl}}(\Omega),
\end{aligned}
$$

we obtain the single-sided noise spectral densities. Here $S_{1}^{(i)}(\Omega)$ is the spectrum of technical input laser noise while $S_{\mathrm{cl}}(\Omega)$ characterizes the spectrum of all the other classicalnoise sources. In further calculations we will assume the laser noise to be white and $10 \mathrm{~dB}$ above the vacuum noise level. For other classical-noise sources, we use the current noise budget of Advanced LIGO, as given in Bench [18]; contributions such as suspension thermal noise, seismic noise, and thermal fluctuations in the coating and gravity gradient noise are presented in Fig. 4.

Note that we can obtain two input-output relations from the equations of motion in Eq. (1)-(5) and write them in the following compact form:

$$
\hat{y}^{(1)}=\vec{n}_{1}^{T} \vec{\nu}+s_{1} h, \quad \hat{y}^{(2)}=\vec{n}_{2}^{T} \vec{\nu}+s_{2} h,
$$

where $\vec{\nu}^{T}=\left(\hat{a}_{1}^{(1)}, \hat{a}_{2}^{(1)}, \hat{a}_{1}^{(2)}, \hat{a}_{2}^{(2)}, \hat{b}_{1}^{(1)}, \hat{b}_{1}^{(2)}, \hat{\xi}_{\mathrm{ITM}}, \hat{\xi}_{\mathrm{ETM}}, \hat{\xi}_{\mathrm{BS}}\right)$ and $T$ denotes transposed. Here the two vectors $\vec{n}_{1,2}$ are the linear transfer functions from the noise channels $\vec{\nu}$ into the two output channels, while the two functions $s_{1,2}$ are the linear transfer functions from the signal, i.e., the $\mathrm{GW}$ strain $h$, into the output channels.

\section{B. Combined sensitivity}

Now we seek for a linear combination of the two output channels, $\hat{y}^{(1)}$ and $\hat{y}^{(2)}$,

$$
\hat{y}=K_{1}(\Omega) \hat{y}^{(1)}+K_{2}(\Omega) \hat{y}^{(2)},
$$

which has optimal sensitivity to gravitational waves. In this optimization, we only consider the signal-referred noise spectral density of $\hat{y}$,

$$
S_{h}(\Omega)=\frac{\left(\begin{array}{ll}
K_{1} & K_{2}
\end{array}\right) \mathbf{N}\left(\begin{array}{l}
K_{1}^{*} \\
K_{2}^{*}
\end{array}\right)}{\left(\begin{array}{ll}
K_{1} & K_{2}
\end{array}\right) \mathbf{S}\left(\begin{array}{l}
K_{1}^{*} \\
K_{2}^{*}
\end{array}\right)}
$$

with 

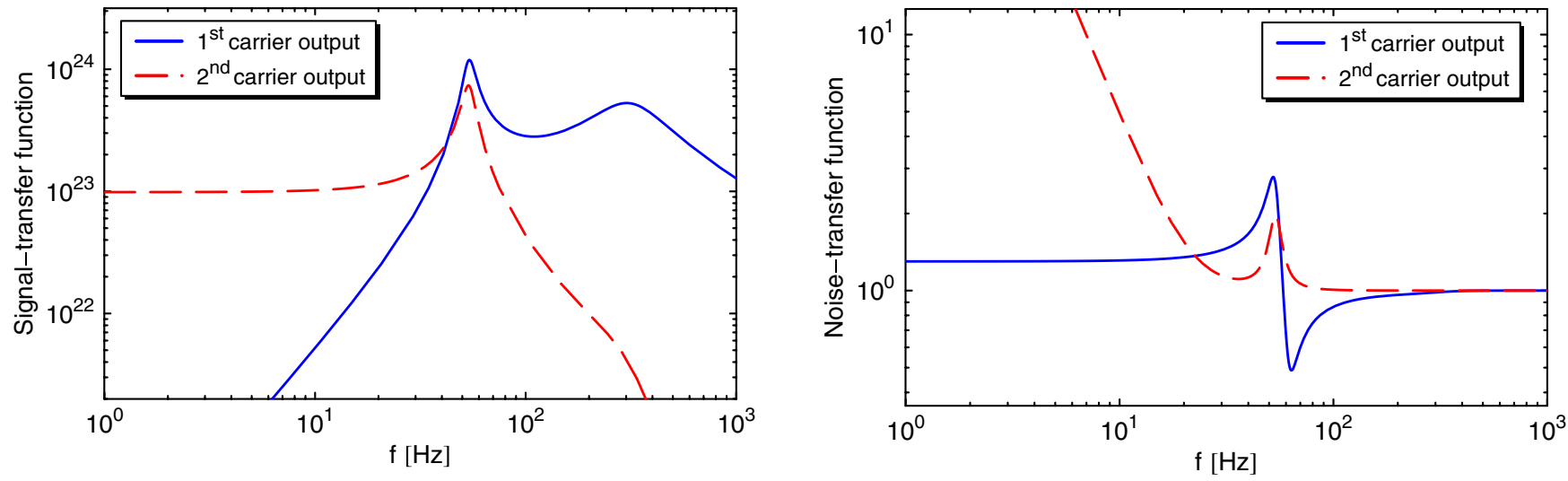

FIG. 2 (color online). Example for the signal (left panel) and noise (right panel) transfer functions in a signal-recycled Michelson interferometer with two carriers and double-readout, for a configuration with the parameters as given in Table I but $\zeta^{(1)}=0$, $\phi=$ $\pi / 2-0.014 \pi$, and $P^{(1)}=800 \mathrm{~kW}$.

$$
\mathbf{N} \equiv\left[\begin{array}{c}
\vec{n}_{1}^{T} \\
\vec{n}_{2}^{T}
\end{array}\right]\left[\begin{array}{lllll}
\mathbb{1}_{4} & & & & \\
& S_{1}^{(1)} & & & \\
& & S_{1}^{(2)} & & \\
& & & 2 S_{\mathrm{cl}} \mathbb{1}_{2} & \\
& & & & S_{\mathrm{cl}}
\end{array}\right]\left[\begin{array}{ll}
\vec{n}_{1}^{*} & \vec{n}_{2}^{*}
\end{array}\right]
$$

and

$$
\mathbf{S} \equiv\left(\begin{array}{ll}
s_{1} s_{1}^{*} & s_{1} s_{2}^{*} \\
s_{2} s_{1}^{*} & s_{2} s_{2}^{*}
\end{array}\right)
$$

where $\mathbb{1}_{k}$ stands for a $k$-dimensional identity matrix. One way of obtaining the minimum noise is to impose the constraint that the value of the denominator always remains unity, and minimize the numerator under this constraint. Note that an overall rescaling of the vector $\left(K_{1}, K_{2}\right)$ does not affect $S_{h}$. The resulting minimum noise is one over the bigger eigenvalue of the 2-by-2 matrix

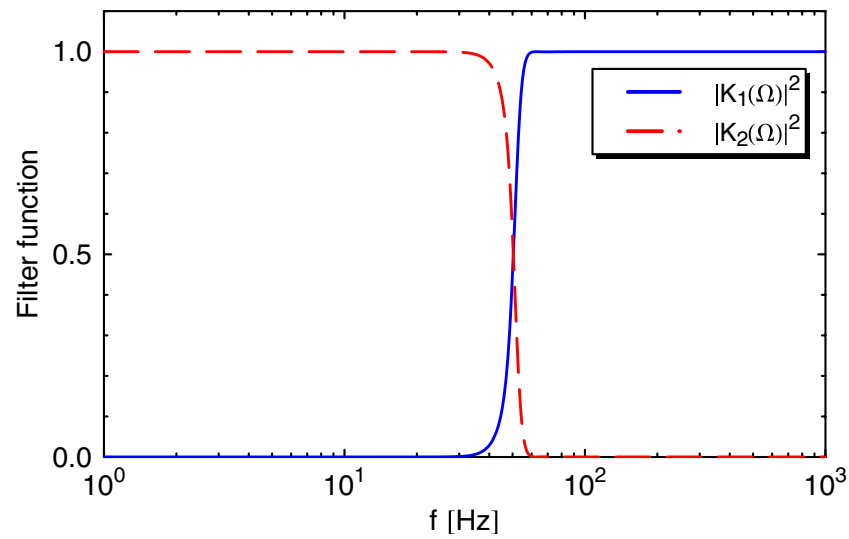

$\mathbf{M} \equiv \mathbf{N}^{-1} \mathbf{S}$

with the corresponding eigenvector providing the optimal filters $\left(K_{1}, K_{2}\right)$.

We now illustrate the local readout scheme using the following configuration: the parameters are given in Table I as well as phase quadrature readout $\zeta^{(1)}=0$, signalrecycling cavity detuning phase $\phi=\pi / 2-0.014 \pi$, and power $P^{(1)}=800 \mathrm{~kW}$ of the first carrier are used. In Fig. 2 we plot the individual signal- and noise-transfer functions of the first and second carriers, for the configuration with $P^{(2)}=4 \mathrm{~kW}$. As we can see from these plots, the first carrier mainly senses frequencies above the optical-spring resonance with signal-transfer function suppressed at lower frequencies by the optical spring; the second carrier offers complementary sensitivity for frequencies below the optical-spring resonance, when the ITM is dragged together with the ETM by the optical spring. As a consequence, as we see in the left panel of Fig. 3, at frequencies

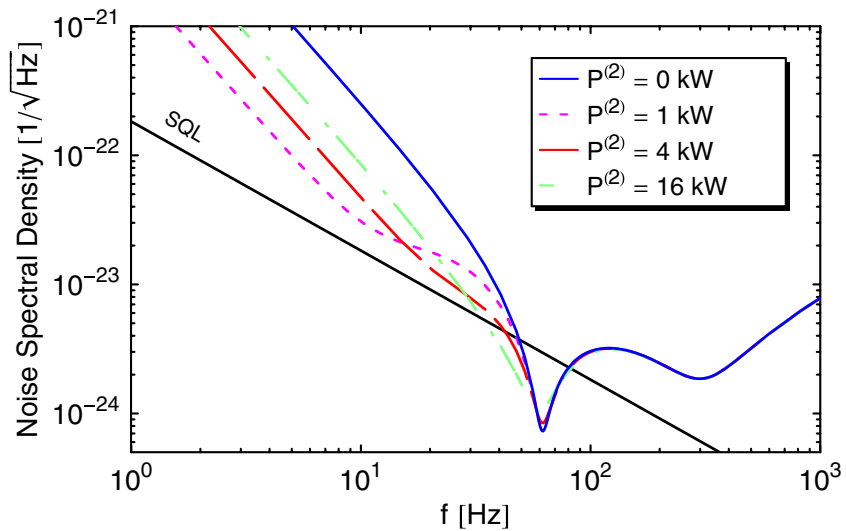

FIG. 3 (color online). Left panel: filter functions $K_{1}$ and $K_{2}$, for the same configuration as in Fig. 2. Here each filter function is rescaled such that it gives the percentage of how much GW strain it feeds into the combined output. Right panel: quantum noise curves for our proposed scheme with different powers of the 2 nd carrier. Again phase quadrature readout $\zeta^{(1)}=0$, signal-recycling cavity detuning phase $\phi=\pi / 2-0.014 \pi$, and power $P^{(1)}=800 \mathrm{~kW}$ of the 1 st carrier are used. 
above the optical-spring resonance, the optimal combination depends mostly on the first readout, while at frequencies below the optical-spring resonance, the optimal combination depends mostly on the second readout.

Noise curves with optimal filters are plotted for different powers of the second carrier $(0 \mathrm{~kW}, 1 \mathrm{~kW}, 4 \mathrm{~kW}$, and $16 \mathrm{~kW}$ ) in the right panel of Fig. 3 where only quantum noise is taken into account. This plot illustrates that the local readout scheme can directly improve the sensitivity only below the optomechanical resonance frequency. It turns out that $4 \mathrm{~kW}$ in each arm of the local meter already gives a remarkable increase in sensitivity. In the following studies we fix $P^{(2)}=4 \mathrm{~kW}$.

One could imagine that the combination of a signalrecycled Michelson interferometer with a local readout may indirectly help improve the sensitivity at high frequencies or increase the detection bandwidth, once an overall optimization to a broadband source is performed. The underlying effect is that the sensitivity of the largescale interferometer can be shifted to higher frequencies by choosing its detection angle to be closer to the phase quadrature while the local meter helps to maintain sensitivity at low frequencies. This will be studied more carefully in Sec. III.

\section{Control}

As it has been shown in Refs. [7-9] the optical spring introduces an instability, which must be stabilized using a feedback control system. In single-readout systems, it is easy to show that such a control system does not give rise to any fundamental change in our GW sensitivity [7-9], intuitively because signal and noise are fed back with the same proportion onto the test masses. Our double-readout system is more complex, but the same intuition still applies. If we denote $\vec{x} \equiv\left(\hat{x}_{\mathrm{ITM}}, \hat{x}_{\mathrm{ETM}}, \hat{x}_{\mathrm{BS}}\right)^{T}$ and $\vec{y} \equiv$ $\left(\hat{y}^{(1)}, \hat{y}^{(2)}\right)^{T}$, the Eqs. (1)-(5) can be written schematically as

$$
\begin{gathered}
\vec{x}=\mathbf{A}(\Omega) \vec{x}+\mathbf{B}(\Omega) \vec{\nu}+\vec{C}(\Omega) h+\mathbf{D}(\Omega) \vec{y}, \\
\vec{y}=\mathbf{F}(\Omega) \vec{x}+\mathbf{G}(\Omega) \vec{\nu} .
\end{gathered}
$$

Here matrix A describes mirror dynamics, matrix $\mathbf{B}$ describes how the noise sources in $\vec{\nu}$ are applied as forces onto the mirrors, vector $\vec{C}$ describes how GW signal $h$ directly influences the mirrors, $\mathbf{F}$ describes how the output channels $\vec{y}$ sense the various motions $\vec{x}$, $\mathbf{G}$ describes sensing noise in $\vec{y}$, and finally $\mathbf{D}$ describes the feedback. Solving Eqs. (13) and (14) jointly, we obtain

$$
\vec{y}=\left[\mathbb{1}_{2}-\mathbf{H D}\right]^{-1}[[\mathbf{H B}+\mathbf{G}] \vec{\nu}+\mathbf{H} \vec{C} h],
$$

where we have defined $\mathbf{H} \equiv \mathbf{F}\left(\mathbb{1}_{2}-\mathbf{A}\right)^{-1}$. In Eq. (15) the only dependence of $\vec{y}$ on the control system is through $\mathbf{D}$, which only appears in the first factor on the right-hand side. The optimal sensitivity, which is obtained by maximizing signal-referred noise spectrum of $\left(K_{1}, K_{2}\right) \vec{y}$, is then clearly invariant with respect to changes in $\mathbf{D}$.

\section{IMPROVEMENTS IN ADVANCED LIGO SENSITIVITY}

\section{A. Matched-filtering signal-to-noise ratio}

To quantify the astrophysical merit of various configurations, we will calculate the improvement in the matchedfiltering signal-to-noise ratio (SNR) or the detectable distance for a given threshold SNR, respectively, for inspiral waves from compact binary systems. For a known waveform (in the frequency domain) $h(f)$, the optimal SNR achievable by correlating the data with a known template is

$$
\rho=2 \sqrt{\int_{0}^{\infty} \mathrm{d} f \frac{|h(f)|^{2}}{S_{h}(f)}}
$$

where $S_{h}(f)$ is the single-sided noise spectral density. For compact binary objects, the lowest post-Newtonian approximation gives (see, e.g., [19])

$$
|h(f)|=\frac{G^{5 / 6} \mu^{1 / 2} M^{1 / 3}}{\sqrt{30} \pi^{2 / 3} c^{3 / 2} D} f^{-7 / 6} \Theta\left(f_{\max }-f\right)
$$

with

$$
M=\left(M_{1}+M_{2}\right) \quad \text { and } \quad \mu=\frac{M_{1} M_{2}}{M_{1}+M_{2}},
$$

where $\mu, M, M_{1}$, and $M_{2}$ are the reduced, total, and single masses of the binary and $D$ is the distance from the source to the detector. Here the amplitude is the one where the rms average over all directions is already taken into account. There is an upper cutoff frequency, $f_{\max }$, in Eq. (17) beyond which the system undergoes a transition from adiabatic inspiral into nonadiabatic merger, and Eq. (17) is no longer a valid approximation. This frequency is usually taken to be the GW frequency at the last stable circular orbit given, for a test mass in a Schwarzschild space-time with mass $M$,

$$
f_{\max } \approx 4400 \mathrm{~Hz}\left(\frac{M_{\odot}}{M}\right) .
$$

A lower cutoff frequency $f_{\min }$ should also be applied to the integration in Eq. (16), below which it is no longer possible to treat the system as stationary. We take $f_{\min } \approx 7 \mathrm{~Hz}$. Considering binaries of averaged orientation the observable distance for a given SNR $\rho_{0}$ reaches

$$
D=\sqrt{\frac{2}{15}} \frac{G^{5 / 6} \mu^{1 / 2} M^{1 / 3}}{\pi^{2 / 3} c^{3 / 2} \rho_{0}} \sqrt{\int_{f_{\min }}^{f_{\max }} \mathrm{d} f \frac{f^{-7 / 3}}{S_{h}(f)}} .
$$

In this paper, we assume event rate to be proportional to the cube of detectable distance, i.e.,

$$
\mathcal{R} \propto D^{3} .
$$




\section{B. Improvement in the event rate}

The tools reviewed in the previous subsection enable us to optimize a specific interferometer configuration for given binary inspirals by maximizing its SNR with respect to certain interferometer parameters. Note that we now also take classical noise into account as is indicated by the gray lines in Fig. 4. In this paper we assume that Advanced LIGO refers to a signal-recycled interferometer without local readout and optimized for neutron-star-neutron-star (NS-NS) binary systems, i.e., binary systems with $M=$ $(1.4+1.4) M_{\odot}$. We then vary the optical power $P^{(1)}$, detuning $\phi^{(1)}$, and detection angle $\zeta^{(1)}$ in such a way that the SNR of the signal-recycled interferometer without local readout is maximized for the total mass of a given binary system in Table II. When we optimize our scheme, we maximize the SNR varying the same set of parameters of the large-scale interferometer (cf. Table II) but with imposing a fixed power for the second carrier $\left(P^{(2)}=4 \mathrm{~kW}\right)$, requiring the second carrier to be resonant in the signalrecycling cavity $\left(\lambda^{(2)}=0\right)$, and fixing a detection quadrature phase of $\zeta^{(2)}=0$ (i.e., detecting the phase quadra- ture). Such a prescription is justified, because a local meter with such a short arm length, low power, and finesse (as we have chosen) is mostly dominated simply by shot noise.

If we compare the two schemes with and without an added local meter at the binary mass they are optimized for we find moderate improvement in event rates (cf. last column in Table II). The improvement increases for higher binary masses since our scheme helps to enhance sensitivity mainly at low frequencies. Such a moderate improvement has been limited mainly due to low-frequency classical noise.

The advantage of the local readout scheme can be appreciated better when we realize that there are different populations of likely sources (e.g., binary total mass $M$ can reside in a range, $\mathcal{M}$ ), whose signals extend to different frequency bands. We need to investigate how well a configuration optimized for a particular system with total mass $M$ would perform for other possible masses in $\mathcal{M}$. In this paper, we consider $\mathcal{M}=\left[M_{\odot}, 630 M_{\odot}\right]$ with maximum mass determined by the condition $f_{\max }=f_{\min }$. In Fig. 5, we show the improvements in event rates (with respect to Advanced LIGO baseline, optimized for NS-NS binaries)

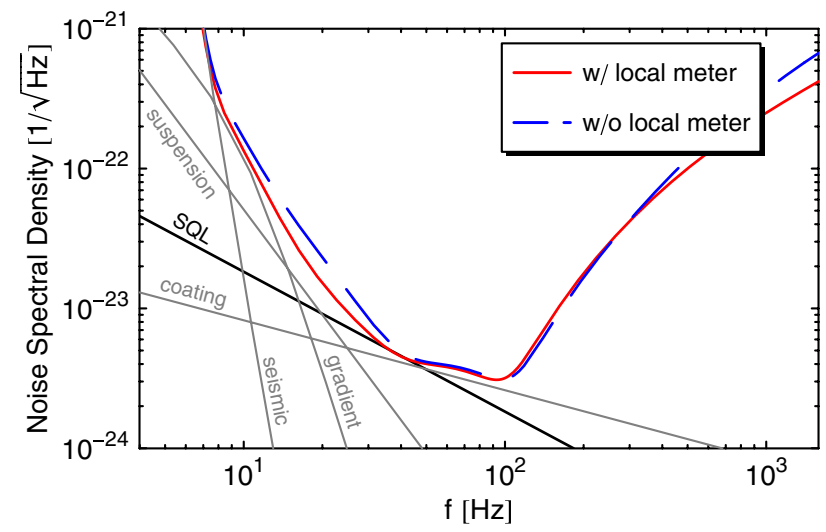

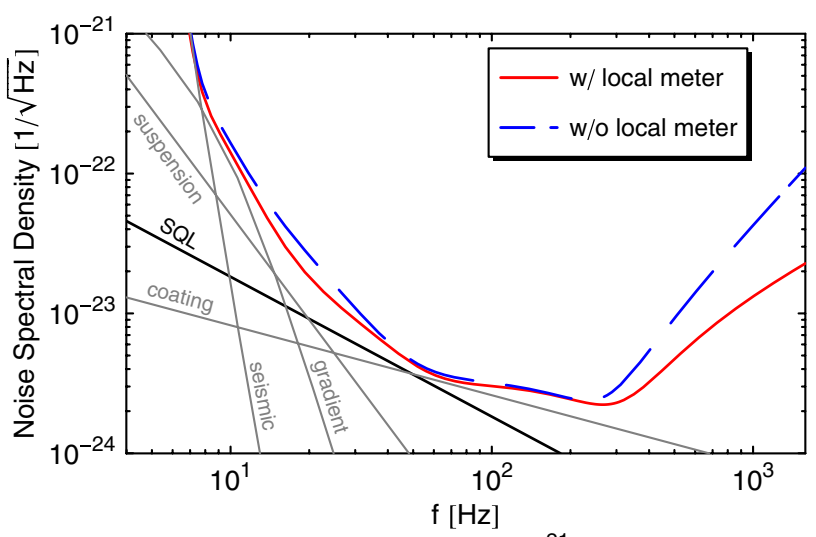

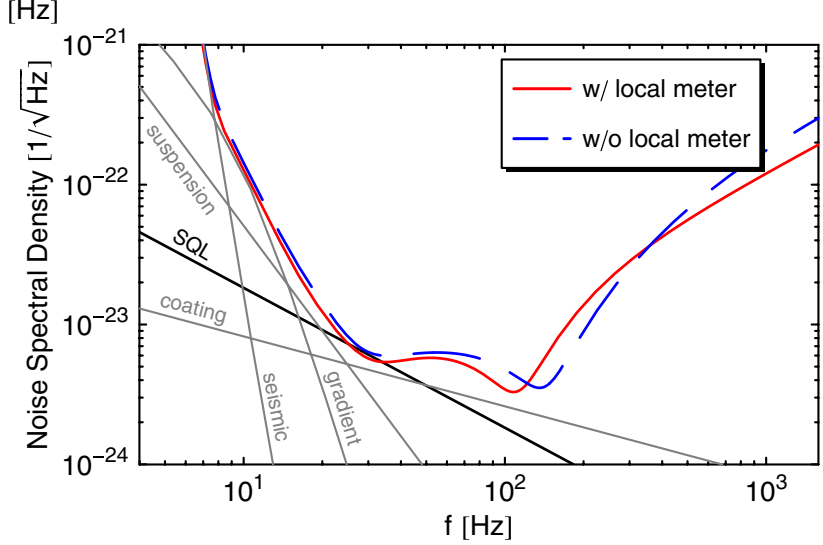

FIG. 4 (color online). Noise curves for the scheme with local readout (power of the 2 nd carrier fixed to $\left.P^{(2)}=4 \mathrm{~kW}\right)$ and without local readout both optimized for binary systems with total mass $M=2.8 M_{\odot}$ (upper left), $M=40 M_{\odot}$ (upper right), and $M=120 M_{\odot}$ (lower). Special parameters used for optimizations are given in Table II and all others in Table I. Here classical noise ( gray lines) is included. Single contributions of the classical- noise are labeled according to their appearance: suspension thermal noise results from the fluctuations in the suspension system; seismic noise is due to motion of the ground; thermal fluctuations in the coating dominates the one in the substrate; gravity gradient noise accounts for time-changing Newtonian gravitational forces. 
TABLE II. Parameters used when optimizing our proposed double-readout scheme and the usual Advanced LIGO-like configuration each for different binary systems. The last column gives the improvement in the event rate for our proposed scheme compared to the usual scheme, both optimized for the given equally distributed total binary mass. Reasonable errors in $\phi$ and $\zeta^{(1)}$ may decrease the event rate-but not more than $1 \%$.

\begin{tabular}{lccccccc}
\hline \hline \multirow{2}{*}{$M / M_{\odot}$} & \multicolumn{2}{c}{ optimization parameters w/ local meter } & \multicolumn{2}{c}{ optimization parameters w/o local meter } & \multirow{2}{*}{ improvement in event rate } \\
\cline { 2 - 6 } & $P^{(1)}$ in $\mathrm{kW}$ & $\phi$ in radian & $\zeta^{(1)}$ in radian & $P^{(1)}$ in $\mathrm{kW}$ & $\phi$ in radian & $\zeta^{(1)}$ in radian & $29 \%$ \\
\hline 2.8 & 800 & $0.48 \pi$ & $0.7 \pi$ & 800 & $0.48 \pi$ & $0.49 \pi$ & $28 \%$ \\
20 & 450 & $0.47 \pi$ & $0.58 \pi$ & 500 & $0.48 \pi$ & $0.48 \pi$ & $30 \%$ \\
30 & 250 & $0.46 \pi$ & $0.46 \pi$ & 200 & $0.46 \pi$ & $0.49 \pi$ & $33 \%$ \\
40 & 150 & $0.45 \pi$ & $0.43 \pi$ & 150 & $0.45 \pi$ & $0.46 \pi$ & $44 \%$ \\
80 & 100 & $0.45 \pi$ & $0.38 \pi$ & 100 & $0.45 \pi$ & $0.46 \pi$ & $42 \%$ \\
120 & 100 & $0.46 \pi$ & $0.32 \pi$ & 100 & $0.47 \pi$ & $0.41 \pi$ & $45 \%$ \\
160 & 110 & $0.47 \pi$ & $0.25 \pi$ & 100 & $0.47 \pi$ & $0.30 \pi$ & $48 \%$ \\
200 & 110 & $0.48 \pi$ & $0.25 \pi$ & 100 & $0.48 \pi$ & $0.27 \pi$ & \\
\hline \hline
\end{tabular}

obtainable by Advanced LIGO configurations (solid lines) and double-readout configurations (dashed lines) for binaries with $M \in \mathcal{M}$, when the configurations are optimized specifically for $M=2.8 M_{\odot}$ (black), $40 M_{\odot}$ (dark gray) and $120 M_{\odot}$ (light gray). In Fig. 4, we show the corresponding noise spectral densities of these configurations, together with classical noise. Figures 4 and 5 provides us with at least two possible applications of the double-readout scheme.

Detector with broader frequency band. The sensitivity of the double-readout configuration optimized for $2.8 M_{\odot}$ systems (solid curve on the upper left panel of Fig. 4) is broader in band and globally better than the baseline design of Advanced LIGO (dashed curve in the same figure), particularly at higher frequencies; this demonstrates that when an overall optimization is performed, the local readout can indirectly improve sensitivity at higher frequen-

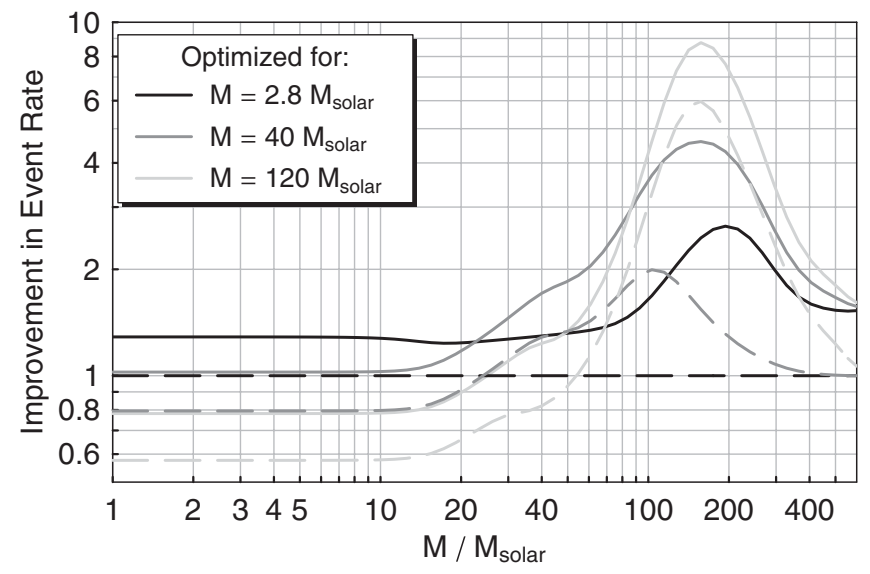

FIG. 5. Improvement in the event rate compared to Advanced LIGO versus total binary mass with fixed optimization parameters for each curve. Signal-recycled interferometer with (solid lines) and without (dashed lines) local readout are optimized for three different binary masses. Power of the 2 nd carrier is fixed to $P^{(2)}=4 \mathrm{~kW}$. cies. Although Fig. 5 (solid curve) does not show a significant increase in binary event rates, this configuration is potentially interesting for detecting other sources above $300 \mathrm{~Hz}$, for example, pulsars, and low-mass x-ray binaries.

Detector for intermediate-mass black-hole binaries. The double-readout configuration optimized for $40 M_{\odot}$ systems (dark gray curve in Fig. 5) has the same sensitivity to low-mass binary systems as Advanced LIGO baseline (up to $M=10 M_{\odot}$ ), while improving event rates for $60 M_{\odot}-300 M_{\odot}$ by factors of $2-4.5$. This allows us to build a detector sensitive to the more speculative (yet in some sense astrophysically more interesting) intermediate-mass black-hole binaries, without sacrificing sensitivity at lowmass systems which are more certain to exist. As we see from dashed curves in Fig. 5, such broad improvement simultaneously for systems with different total masses is not achievable by single-readout Advanced LIGO-like configurations. It is also interesting to note that this configuration only requires a circulating power of $150 \mathrm{~kW}$ in the arms.

The improvement in event rate increases significantly for higher binary masses (cf. gray curve in Fig. 5 optimized for $M=120 M_{\odot}$ ) since the local meter helps to enhance sensitivity mainly at low frequencies. But if we optimize for such high masses the sensitivity for lower masses cannot keep up with Advanced LIGO.

It turns out that our scheme even improves sensitivity in the low-frequency regime when sensitivity is dominated by classical noise, as can be seen in Fig. 5, since for high binary masses the dashed curves meet at a factor of $(4 / 3)^{3 / 2}$ below the solid curves. We explain this factor in the appendix.

\section{IMPLEMENTATION ISSUES}

In this section we discuss the possibility of implementing this technique explicitly in the Advanced LIGO detector. In fact, the so-called central Michelson degree of freedom in the detector, already to be measured to keep 
the signal-extraction port of the interferometer in dark fringe, is exactly what our local readout scheme proposes to measure. However, sensitivity of the current Michelson control signal must be improved dramatically in order to be turned into our regime. We note that more precise measurement of this Michelson degree of freedom also helps to decrease control-loop noise, which is shot noise imposed on the control signal coupling to the main signal due to unavoidable imbalances [20].

Optical Power. In the baseline design, a pair of radio frequency (RF) sidebands created around the main carrier frequency will be injected to probe the motion as is already done in current detectors. However, the power level of current RF sidebands is not high enough for our local readout. In the baseline design, the input power is $125 \mathrm{~W}$, which is amplified to $\sim 1.0 \mathrm{~kW}$ at each ITM, due to power recycling. Only about $1 \%$ of the power at the input port is pumped into the RF sidebands that resonate in the powerrecycling cavity but not in the arms. Taking into account the fact that the RF sidebands do not enter the arm cavities and thus suffer from less optical losses, the power of the Michelson-control sidebands at the ITM is currently planned to be $\sim 34 \mathrm{~W}$. Thus, one needs to raise the current power by $\sim 120$ times in order to achieve $P^{(2)}=4 \mathrm{~kW}$. Another more realistic way of realization is to use a phaselocked secondary laser with its frequency shifted by an odd number of half free-spectral ranges from the primary laser to satisfy the off-resonant condition in the arms. Furthermore, this subcarrier should almost be in dark fringe at the signal-extraction port and should be resonant in both recycling cavities. To achieve a circulating power of $P^{(2)}=4 \mathrm{~kW}$ for the subcarrier we even need a little more input power than for the primary laser. But we can hope to use the higher-power laser for the subcarrier while the parametric instability [21,22] in the arm cavity may limit the power of the primary laser. Indeed, a circulating power of $P^{(2)}=4 \mathrm{~kW}$ is only a few times more than the carrier power of the current GEO detector which has a similar topology compared to the local meter.

Detection. Each signal at the dark port should be extracted with some reference field, which will be another set of RF sidebands in the RF readout scheme, or DC offset light in the DC readout scheme. The former one leaks through the dark port via macroscopic asymmetry in the central Michelson interferometer, and the latter one leaks through the dark port via microscopic asymmetry between the two arm cavities. Either way, the reference fields for the carrier and the subcarrier should be isolated before the photo-detection, otherwise the reference field which is not used for the signal extraction will just impose extra shot noise. One way to solve the problem is to make use of orthogonal polarizations. Before the photo-detection, the carrier and the subcarrier accompanied with the reference fields can be separated by a polarized beam splitter, which is all-reflective to one polarization and transmissive to the other. In addition, it is easy to combine the two beams before injection into the interferometer without losing the power. An alternative way to the orthogonal polarizations is to use a cavity that can separate the beams at different frequencies, where one resonates in the cavity while the other does not. The cavity, a so-called output-modecleaner, is already planned to be used at the detection port in Advanced LIGO. In the same way an input mode cleaner cavity can be used to combine two beams before the injection into the interferometer.

Alternative configuration. One may also place the local meters around the ETMs. In this case, a single laser beam, which can be different in frequency from the carrier light, should be split and brought to each end of the arms so that laser noise can be canceled out after taking a subtraction of the two ETMs' motion measurements. A cavity can be implemented as well as it is proposed for a radiationpressure-noise reduction method in $[13,14]$. In this way the secondary laser for the local readout does not need such high power and there is no concern of a heat problem at the BS and the ITMs. However, in this case much more additional optical components are required to realize this configuration.

\section{CONCLUSION}

Motivated by the optical-bar schemes [12] and quantumlocking schemes $[13,14]$, we have proposed injecting a second laser beam into detuned signal-recycled Michelson interferometers, sensing the differential motion of the input mirrors, and improving low-frequency sensitivities of these interferometers, currently at low frequencies being limited by the rigidity of the optical spring. We derived the optimal combined sensitivity of this doublereadout scheme, and demonstrated that this optimal sensitivity is invariant with respect to the application of a feedback control scheme.

Taking into account the current classical-noise budget of Advanced LIGO, as well as constraints on optical power, we performed an optimization of our double-readout schemes toward the detection of compact-binary inspirals. This scheme is shown either to be able to broaden the detection band and (indirectly) significantly improve high-frequency sensitivities, or to allow the detection of intermediate-mass black-hole binaries with a broad frequency range without sacrificing sensitivity to neutronstar binaries and stellar-mass black-hole binaries.

We also discussed briefly how the sensing of the Michelson degree of freedom in the current plan of Advanced LIGO can be made dramatically more sensitive and turned into our local readout scheme.

Finally, we would like to point out that this scheme should be further investigated as a candidate design for third-generation detectors, possibly in conjunction with the injection of squeezed vacuum states $[23,24]$ into the interferometer's dark port $[25,26]$. 


\section{ACKNOWLEDGMENTS}

We thank Stan Whitcomb for very useful discussions. Research of H. M.-E., K. S., and Y.C. is supported by the Alexander von Humboldt Foundation's Sofja Kovalevskaja Programme. Research of H. R. and R. S. is supported by the Deutsche Forschungsgemeinschaft through the EGC program and the SFB No. 407, respectively. Research of C. L. is supported in part by NSF grants No. PHY-0099568 and No. PHY-0601459.

\section{APPENDIX A: DOUBLE-READOUT SCHEME DOMINATED BY CLASSICAL NOISE}

Suppose at low frequencies, sensing noise is negligible, and noise is dominated by the classical force noise acting on the mirrors. Then, the first carrier offers the following:

$$
\hat{y}^{(1)} \propto \hat{\xi}_{\mathrm{ETM}}-\hat{\xi}_{\mathrm{ITM}}+L h,
$$

where $\xi_{\text {ETM }}$ and $\xi_{\text {ITM }}$ are classical noise on the ETM and the ITM, respectively. The output of the second carrier is proportional to

$$
\hat{y}^{(2)} \propto \hat{\xi}_{\mathrm{ETM}}+\hat{\xi}_{\mathrm{ITM}}+2 \sqrt{2} \hat{\xi}_{\mathrm{BS}}+L h,
$$

where $\xi_{\mathrm{BS}}$ is the classical noise acting on the BS. Suppose again that $\xi_{\mathrm{ITM}}, \xi_{\mathrm{ETM}}$, and $\xi_{\mathrm{BS}}$ have independent noise at the same level for ITM and ETM but half as high for the BS [cf. Eq. (6)]. We obtain that the optimal filter uses $3 / 4$ of the output of the large-scale interferometer and $1 / 4$ of the small interferometer in the units as above. This is in contrast to the optimal filter functions when only quantum noise is taken into account as in the left panel of Fig. 3. Then the combined output is given by

$$
\hat{y} \propto \xi_{\mathrm{ETM}}-\frac{1}{2} \xi_{\mathrm{ITM}}+\frac{1}{\sqrt{2}} \xi_{\mathrm{BS}}+L h .
$$

Then the large-scale interferometer's noise spectral density versus the optimal noise spectral density reads $2 / \frac{3}{2}$ which gives the factor in Fig. 5. In this way the double-readout is able to cancel some fraction of the classical noise.
[1] D. Shoemaker (LIGO Scientific Collaboration), Nucl. Instrum. Methods Phys. Res., Sect. A 517, 154 (2004).

[2] L. D. Fiore (VIRGO collaboration), Classical Quantum Gravity 19, 1421 (2002).

[3] B. Willke et al., Classical Quantum Gravity 19, 1377 (2002).

[4] M. Ando (TAMA collaboration), Phys. Rev. Lett. 86, 3950 (2001).

[5] G. Heinzel, A. Freise, H. Grote, K. Strain, and K. Danzmann, Classical Quantum Gravity 19, 1547 (2002).

[6] Advanced LIGO Reference Design, LIGO M060056, http://www.ligo.caltech.edu/docs/M/M060056-07/ M060056-07.pdf.

[7] A. Buonanno and Y. Chen, Phys. Rev. D 64, 042006 (2001).

[8] A. Buonanno and Y. Chen, Phys. Rev. D 65, 042001 (2002).

[9] A. Buonanno and Y. Chen, Phys. Rev. D 67, 062002 (2003).

[10] K. Somiya, P. Beyersdorf, K. Arai, S. Sato, S. Kawamura, O. Miyakawa, F. Kawazoe, S. Sakata, A. Sekido, and N. Mio, Appl. Opt. 44, 3179 (2005).

[11] O. Miyakawa et al., Phys. Rev. D 74, 022001 (2006).

[12] V. B. Braginsky, M. L. Gorodetsky, and F. Y. Khalili, Phys. Lett. A 232, 340 (1997).

[13] S. Kawamura, O. Miyakawa, and K. Somiya, "Reduction of Radiation Pressure Noise Using an Auxiliary Interferometer in a Laser Interferometric Gravitational Wave Antenna" (unpublished).
[14] J. M. Courty, A. Heidmann, and M. Pinard, Phys. Rev. Lett. 90, 083601 (2003).

[15] H. J. Kimble, Y. Levin, A. B. Matsko, K. S. Thorne, and S. P. Vyatchanin, Phys. Rev. D 65, 022002 (2001).

[16] T. Corbitt, Y. Chen, and N. Mavalvala, Phys. Rev. A 72, 013818 (2005).

[17] J. Harms, R. Schnabel, and K. Danzmann, Phys. Rev. D 70, 102001 (2004).

[18] http://www.ligo.mit.edu/bench/bench.html.

[19] T. Damour, B. R. Iyer, and B. S. Sathyaprakash, Phys. Rev. D 62, 084036 (2000).

[20] K. Somiya, O. Miyakawa, P. Fritschel, and R. Adhikali, Report No. LIGO-T060272-00-I, 2006.

[21] V. B. Braginsky, S. E. Strigin, and S. P. Vyatchanin, Phys. Lett. A 287, 331 (2001).

[22] V. B. Braginsky, S. E. Strigin, and S. P. Vyatchanin, Phys. Lett. A 305, 111 (2002).

[23] H. Vahlbruch, S. Chelkowski, B. Hage, A. Franzen, K. Danzmann, and R. Schnabel, Phys. Rev. Lett. 97, 011101 (2006).

[24] K. McKenzie, N. Grosse, W. P. Bowen, S. E. Whitcomb, M. B. Gray, D. E. McClelland, and P. K. Lam, Phys. Rev. Lett. 93, 161105 (2004).

[25] J. Harms, Y. Chen, S. Chelkowski, A. Franzen, H. Vahlbruch, K. Danzmann, and R. Schnabel, Phys. Rev. D 68, 042001 (2003).

[26] A. Buonanno and Y. Chen, Phys. Rev. D 69, 102004 (2004). 